\title{
Spontaneous fluctuations in a magnetic Fe/Gd skyrmion lattice
}

\author{
M. H. Seaberg, ${ }^{1}$ B. Holladay, ${ }^{1,2}$ S. A. Montoya,${ }^{3,4}$ X. Y. Zheng, ${ }^{1}$ J. C. T. Lee,${ }^{5}$ A. H. Reid, ${ }^{1}$ J. D. Koralek, ${ }^{1}$ L. Shen, ${ }^{6}$ \\ V. Esposito, ${ }^{6}$ G. Coslovich, ${ }^{1}$ P. Walter, ${ }^{1}$ S. Zohar, ${ }^{1}$ V. Thampy, ${ }^{1}$ M. F. Lin, ${ }^{1}$ P. Hart, ${ }^{1}$ K. Nakahara, ${ }^{1}$ R. Streubel,,${ }^{7,8}$ \\ S. D. Kevan, ${ }^{5}$ P. Fischer, ${ }^{7,9}$ W. Colocho, ${ }^{1}$ A. Lutman, ${ }^{1}$ F.-J. Decker, ${ }^{1}$ E. E. Fullerton, ${ }^{4,10}$ M. Dunne, ${ }^{1}$ \\ S. Roy, ${ }^{5}$ S. K. Sinha, ${ }^{2}$ and J. J. Turner $\oplus^{1,6, *}$ \\ ${ }^{1}$ Linac Coherent Light Source, SLAC National Accelerator Laboratory, Menlo Park, California 94720, USA \\ ${ }^{2}$ Department of Physics, University of California-San Diego, La Jolla, California 92093, USA \\ ${ }^{3}$ Naval Information Warfare Center Pacific, San Diego, California 92152, USA \\ ${ }^{4}$ Center for Memory and Recording Research, University of California-San Diego, La Jolla, California 92093, USA \\ ${ }^{5}$ Advanced Light Source, Lawrence Berkeley National Laboratory, Berkeley, California 94720, USA \\ ${ }^{6}$ Stanford Institute for Materials and Energy Sciences, Stanford University and SLAC National Accelerator Laboratory, Menlo Park, \\ California 94025, USA \\ ${ }^{7}$ Materials Sciences Division, Lawrence Berkeley National Laboratory, Berkeley, California 94720, USA \\ ${ }^{8}$ Department of Physics \& Astronomy and Nebraska Center for Materials and Nanoscience, University of Nebraska-Lincoln, Lincoln, \\ Nebraska 68588, USA \\ ${ }^{9}$ Physics Department, UC Santa Cruz, Santa Cruz, California 95064, USA \\ ${ }^{10}$ Department of Electrical and Computer Engineering, University of California-San Diego, La Jolla, California 92093, USA
}

(Received 8 February 2021; revised 29 April 2021; accepted 19 August 2021; published 15 September 2021)

\begin{abstract}
Magnetic skyrmions are topological spin textures that exhibit classical or quantum quasiparticle behavior. A substantial amount of research has occurred in this field, both because of their unique electromagnetic properties and potential application for future nonvolatile memory storage applications, as well as fundamental questions on their topology and unique magnetic phases. Here, we investigate the fluctuation properties of a magnetic $\mathrm{Fe} / \mathrm{Gd}$ skyrmion lattice, using short-pulsed $\mathrm{x}$ rays. We first measure spontaneous fluctuations of the skyrmion lattice phase and find an inherent, collective mode showing an underdamped oscillation with a relaxation of a couple of nanoseconds. Further observations track the response towards the continuous phase transition and a "critical-like" slowing down of fluctuations is observed well before the critical point. These results suggest that the skyrmion lattice phase never fully freezes into a static crystal. This constant state of fluctuation indicates that the physics of topological magnetic phases may have more in common with high-temperature superconductors with disorder.
\end{abstract}

DOI: 10.1103/PhysRevResearch.3.033249

\section{INTRODUCTION}

Skyrmions represent one of the most complex forms of magnetic order yet observed: They consist of a localized excitation, constructed from a vortexlike, continuous rotation of magnetic spins, which represents a topological quasiparticle. These objects are proposed as interesting candidates for future information technologies due to their topological protection, mobility, size, and energy consumption compared to domain walls or other conventional technologies [1,2]. The spontaneous creation of these solitons occurs due to a delicate energy balance and allows for the formation of new types of order.

An active focus recently in condensed matter has been on the motion of magnetic skyrmions. Numerous studies have

\footnotetext{
*joshuat@slac.stanford.edu

Published by the American Physical Society under the terms of the Creative Commons Attribution 4.0 International license. Further distribution of this work must maintain attribution to the author(s) and the published article's title, journal citation, and DOI.
}

demonstrated that skyrmions can be displaced with electrical currents with low-power consumption [3-6]. A recent highlight is in the study of biskyrmions. These are bound pairs of opposite helicity skyrmions, or $|Z|=2$ solitons, where the topological quantum number, also known as the winding number, is $Z= \pm 1$ for each skyrmion of the bound pair [7]. Recent evidence has suggested that a similar lattice state can form which resembles the biskyrmion lattice, but consists of either the $Z=0$ skyrmion bubble states [8], or the single $Z=2$ skyrmion states [9]. Since the precise topology of these is still being actively debated, we simply refer to them here as "skyrmion lattice states." These structures have been heavily studied due to the observation that they can be electrically driven by orders of magnitude lower current density [10-13]. However, an outstanding question is how do spontaneous fluctuations affect the formation of topological magnetic phases (e.g., skyrmions, biskyrmions, etc.) and the transition to other magnetic phases?

Fluctuations are critical for attempting to understand the stability of this phase, the microscopic interactions among the constituents of this phase, and their role in the response functions of the system, yet little has been explored in this 
area. Important for understanding skyrmion physics is how the skyrmion-skyrmion interaction, as well as the interaction between skyrmion bound pairs, manifests in the formation of the skyrmion lattice state. The short length scale for these interactions signifies the importance of implementing a probe sensitive to this length scale, such as the case with neutron or $\mathrm{x}$-ray scattering.

In this paper, we address these open questions through an investigation of stochastic fluctuations by studying a roomtemperature skyrmion $\mathrm{Fe} / \mathrm{Gd}$ multilayer specimen. This is accomplished by illuminating the system with ultrashort pairs of $\mathrm{x}$-ray pulses generated at an $\mathrm{x}$-ray free-electron laser (X-FEL) [14] by using two electron bunches as the $\mathrm{x}$-ray pair source [15]. In contrast to other scattering techniques which measure an average structure, here subtle differences of the magnetic configuration-which change in time between the pulses - can be measured using the resultant coherent diffraction pattern from the sample, differences which are encoded in the $\mathrm{x}$-ray speckle structure [16]. Based on the degree of fluctuation of the sample occurring between the two time-varied $\mathrm{x}$-ray pulses, the dynamics of the system can be measured directly as shown recently [17]. The changes are measured by formulating a probability distribution from counting individual photons, and using speckle statistics to extract the normalized intermediate scattering function $S(q, t)$. By probing the magnetic state formed after the skyrmions coalesce into a lattice state, we found evidence of highly dynamic fluctuations. These slow down with field, well before the critical point separating the uniform ferromagnetic phase, providing evidence of a skyrmion lattice state which never fully freezes. Most importantly, where the lattice state is most rigid, we nonetheless observe an underdamped, propagating excitation of the skyrmion lattice.

\section{EXPERIMENTAL}

The sample measured was a thin-film $\mathrm{Fe} / \mathrm{Gd}$ multilayer with 100 repetitions of alternating $\mathrm{Fe}(0.34 \mathrm{~nm})$ and Gd $(0.4 \mathrm{~nm})$ grown by dc magnetron sputtering $[11,12]$. This system forms a skyrmion lattice at room temperature $[18,19]$. The data presented here were measured at the SXR instrument $[20,21]$ of the Linac Coherent Light Source [14,22] using a newly constructed endstation. This station consists of a UHV chamber housing a sample holder, electromagnet, and megapixel fast readout detector placed $2.35 \mathrm{~m}$ from the sample $[23,24]$ in a forward scattering geometry. The photon energy was set to $1190 \mathrm{eV}$ ( $\mathrm{Gd} M_{5}$ edge) using a grating monochromator [25], a $1.0 \mathrm{eV}$ bandwidth [26,27], and a spot size of $30 \mu \mathrm{m}[28,29]$.

\section{RESULTS}

Figure 1(a) illustrates the magnetic field-temperature phase diagram for a similar specimen, and has been mapped out by x-ray scattering, microscopy, and Lorentz transmission electron microscopy (LTEM), as reported by Montoya et al. [11]. It establishes the number of complex phases which form in this system, and indicates the region of $H-T$ space where the skyrmion lattice forms (yellow region) $[18,30]$. We performed coherent resonant soft x-ray scattering at the $\mathrm{Gd}$
$M_{5}$ edge which is sensitive to the order parameter of the magnetic structure. An example of the diffraction pattern is shown in Fig. 1(b), with the integrated intensity of the symmetric skyrmion lattice peaks with field measured at the X-FEL shown in Fig. 1(c).

We first conducted measurements where the resonant scattering intensity is maximized in the lattice phase, at the skyrmion Bragg reflection at $Q_{s}=6.3 \mathrm{~m}^{-1}$. This occurs at a magnetic field of $H=210 \mathrm{mT}$ [Fig. 1(c)], or a reduced field of $|h|=0.22$, defined as $h=\left(H-H_{c}\right) / H_{c}$ for the critical field $H_{c}=267 \mathrm{mT}$, where the skyrmion lattice melts. This point is where one would expect the state to be the most robust. We observe that this is generally the case, since the structure only partially fluctuates at this timescale, but notably, the state is not fully static.

The intermediate scattering function $S(q, t)$ is shown in Fig. 2(a) which illustrates the skyrmion fluctuations under these conditions, with evidence of an underdamped oscillation of the correlation function occurring at a frequency of $f=$ $0.25 \mathrm{GHz}$. This fits an oscillatory exponential decay, with a relaxation time of $\tau \sim 1.8 \mathrm{~ns}$. The data are normalized by the single-shot data which represent $S(q, 0)$, as has been implemented previously [13]. At this timescale, the contrast decay value indicates that about $75 \%$ of the global skyrmion structure at this point in the phase diagram is moving slower than what these measurements can capture. The lifetime measured here of the skyrmion lattice represents a direct examination of spontaneous fluctuations and indicates rapid thermal motion.

The $S(q, t)$ attained above can be computed by solving for the degree of freedom $M$ using the well-known transcendental equation for photon counting in speckle experiments [31],

$$
P(k)=\frac{\bar{k}^{k}}{k !} \frac{\Gamma(k+M)}{\Gamma(1+M)} M^{M+1}\left(\frac{1}{\bar{k}+M}\right)^{k+M} .
$$

Since $M$ is equal to the inverse of the square of the contrast, it has been shown that the normalized contrast is equal to the normalized intensity-intensity correlation function [32]. Here, $k$ is the number of photons per speckle and $\bar{k}$ is the average intensity. To verify that we are not creating unwanted excitations with the $x$ rays, Fig. 2(b) shows the contrast as a function of the pulse energy of the first pulse [33].

Because Siegert's relation is usually implemented [34], where the contrast goes as the first-order correlation function squared, $g_{2}(q, t)=1+\left|g_{1}(q, t)\right|^{2}$, an oscillation in this type of measurement typically would have the form of $\sim \cos ^{2}(\omega t)$. A pure cosine dependence as observed in Fig. 2(a) can occur however when there is a constant velocity motion within the sampled volume, if two separate velocities are present $v_{1}$ and $v_{2}$ which would exhibit a difference frequency mode, i.e., $\cos \left[\boldsymbol{q} \cdot\left(\boldsymbol{v}_{1}-\boldsymbol{v}_{2}\right) t\right]$, or if a static signal is formulated which interferes with the fluctuating signal. The latter is called "heterodyne" detection and is well known in the photon correlation spectroscopy community [35].

While no velocity gradient evidence is present in this experiment, two modes have been measured for $q=0$, at 1.3 and $1.8 \mathrm{GHz}$ [12], and give a difference within a factor of 2 of what we measure here. However, for $q=Q_{s}$ measured here, we would expect these to occur at higher frequencies. Since we Shannon-sample at $1.4 \mathrm{GHz}$, this could also be a folded 

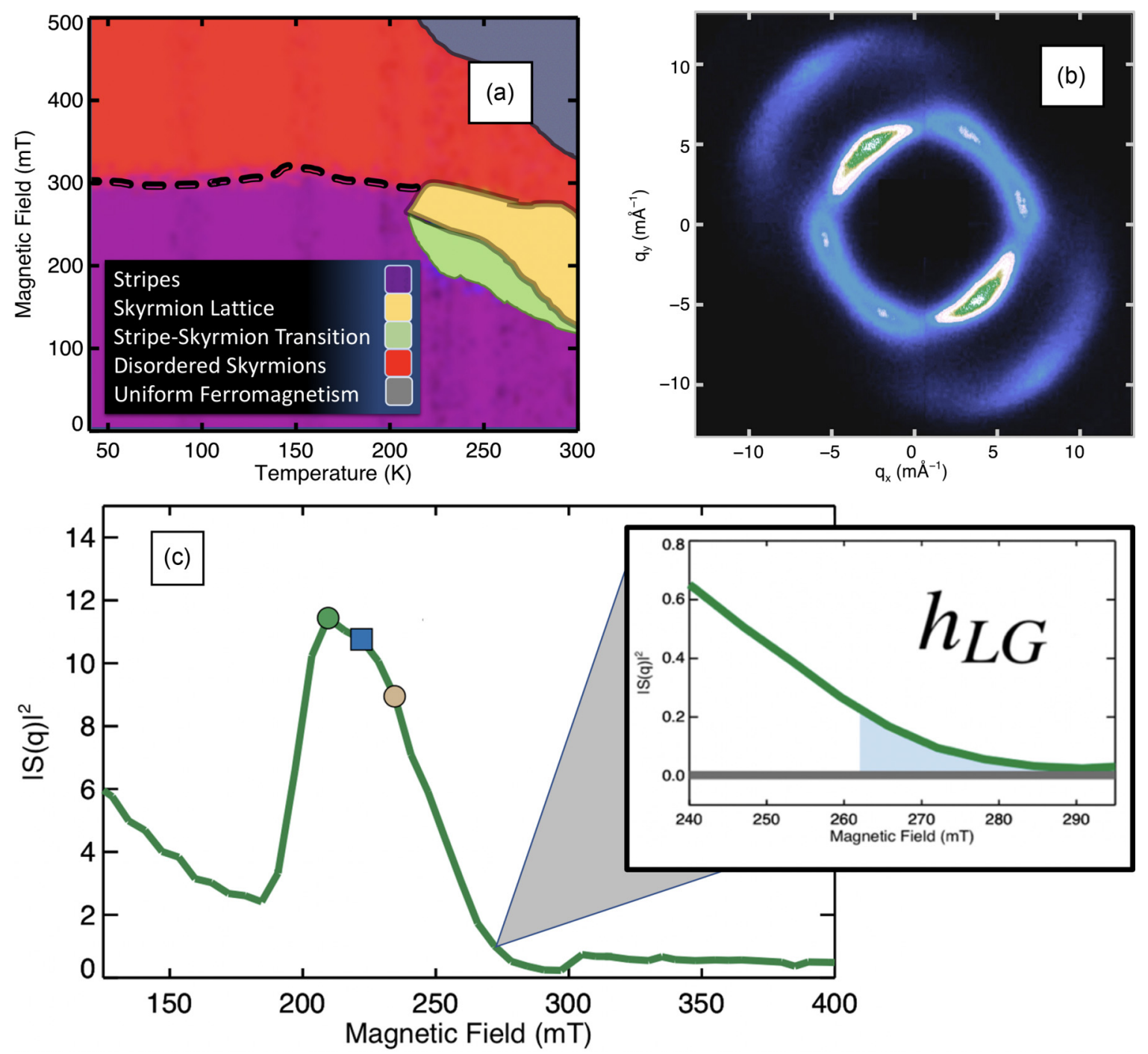

FIG. 1. The skyrmion lattice phase. (a) The magnetic field-temperature phase diagram of Fe/Gd [11]. (b) The average resonant scattering of the biskyrmion lattice phase right as the lattice solidifies $(195 \mathrm{mT})$, time-averaged over about $40 \mathrm{~s}$, or about $4.7 \times 10^{3} \mathrm{x}$-ray pulse pairs. The symmetry breaking of the sixfold symmetric pattern is caused by the biskyrmion units, or bound skyrmion pairs. (c) $S(q, 0)$ for the skyrmion lattice order at $q=Q_{s}$, measured with resonant soft x-ray scattering tuned to the Gd $M_{5}$ edge. The integral of the intensity over the symmetrical skyrmion peaks only is shown with magnetic field, demonstrating the magnetic field conditions to create a stable skyrmion lattice phase at room temperature. The inset in (c) shows a closeup of the plot near the phase transition and designates the calculated Levanyuk-Ginzburg region (blue area), discussed below.

mode, representing another $q=0$ mode at $2.6 \mathrm{GHz}$ [12], but this higher-energy mode would be even more well-separated from the others.

It is possible, however, to have a special case of this scenario, which is based on two opposing velocities in the sample, which would contribute $v_{1}$ and $-v_{1}$, and so create an oscillatory function of twice the frequency, $\sim \cos \left[\boldsymbol{q} \cdot\left(2 \boldsymbol{v}_{1}\right) t\right]$. A recent real-space Brownian motion study has shown that the helicity of skyrmions distinctively affects their thermal motion [36]. Since the lattice consists of bound pairs of opposite helicity skyrmions, we could be measuring the difference between the rotating modes. This phenomenon has for instance been seen in x-ray photon correlation studies of nanoparticle diffusion in polymer melt networks, where opposing ballistic particle motion was observed in one dimension (1D)—along the direction of the chain [37]. For the measured clockwise and counterclockwise rotational modes at the $\sim 1 \mathrm{GHz}$ level and above however [38-40], these would give frequencies an order of magnitude larger than what we measure here.

More likely in this case, and a scenario which would explain the low-energy mode, is the third possibility based on heterodyne detection. This occurs when static and fluctuating signals are mixed at the detector. Since the data taken at the brighter, twofold symmetric skyrmion Bragg peak consists of intensity from both the skyrmion lattice as well as the paired skyrmion objects, a static signal could originate from the increased asymmetrical scattering due to the bound skyrmion pair itself. If the skyrmion-skyrmion bond which couples this skyrmion pair together is more robust than the skyrmion lattice interaction between paired objects, the pair could act as a single, rigid object, only fluctuating with 

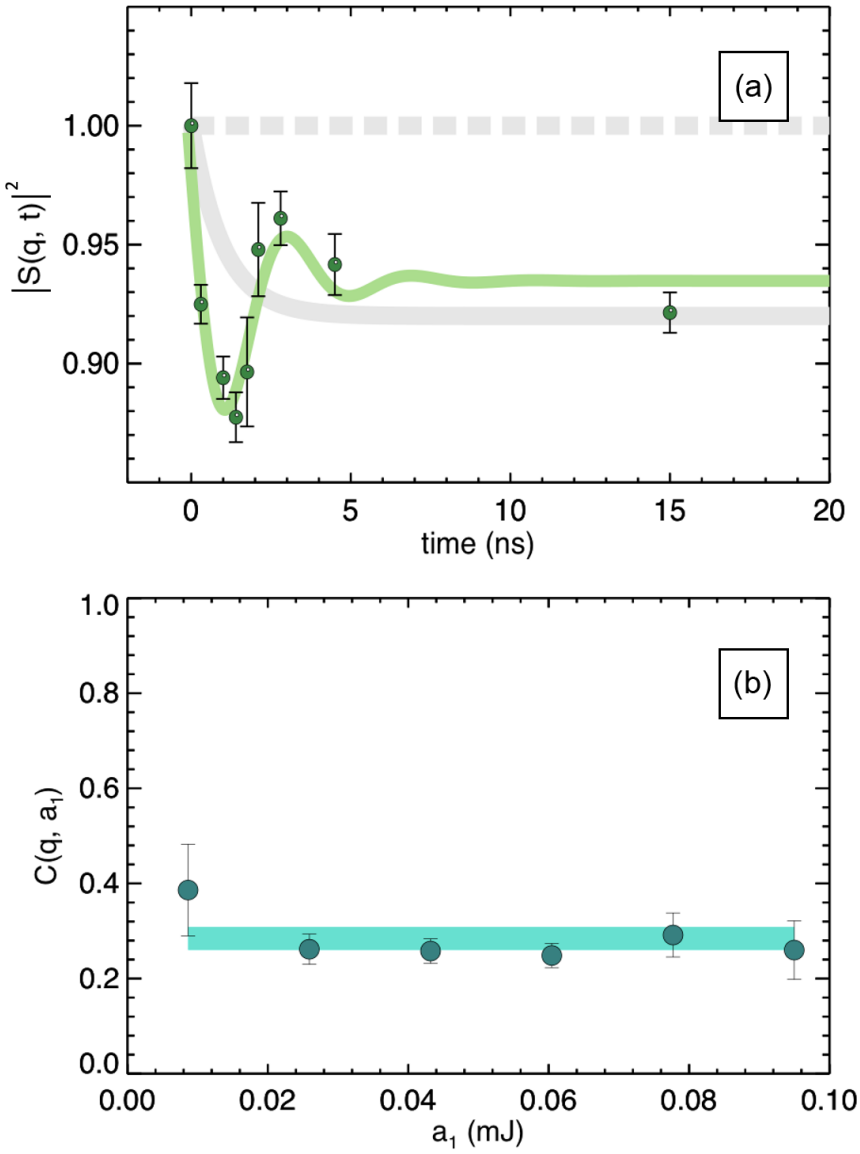

FIG. 2. The skyrmion low-energy mode in Fe/Gd. (a) The normalized $S(q, t)$ shown here for the skyrmion lattice peaks where $q$ is given by $Q_{s}$ for a field of $H=210 \mathrm{mT}$ or $|h|=0.22$ [green circle in Fig. 1(c)]. A damped oscillatory decay is observed for a low-energy mode of the skyrmion lattice for $f=0.25 \mathrm{GHz}$. (b) The contrast data are evaluated for the time separation of $1.0 \mathrm{~ns}$ and plotted as a function of the pulse energy of the first pulse, amplitude $a_{1}$. This curve $C\left(q, a_{1}\right)$ shows no systematic changes with increasing pulse energy.

respect to other paired units in the lattice. This would create an internal heterodyne detection scheme, and allow us to capture the low-energy mode directly. Note that a static bond here means that an internal vibration at higher frequency than the Shannon limit would not be resolved. Further verification of this scenario is the fact that the difference of the intensity between symmetrical (sixfold) and asymmetrical (twofold) peaks does not vanish before the phase transition to the disordered state with magnetic field. This means the structure of the skyrmion speckle pattern is preserved up to the transition, i.e., the disordered state is a fluid of bound skyrmion pairs, rather than separate skyrmions. This indicates that the skyrmion lattice is more susceptible to thermal fluctuations than the more robust, skyrmion-skyrmion bound pair. Finally, a Goldstone mode exists for this lattice which softens as $q \rightarrow$ 0 and has been studied for these types of systems theoretically [41,42]. The finite $q$ measured here would be expected to be at sub-GHz frequencies for the skyrmion wave vector and is consistent with the observations reported here.
To further map how the fluctuations change within the skyrmion lattice phase, we measure at reduced fields of $|h|=$ 0.18 and $|h|=0.12$, closer to the continuous phase transition at $|h|=0$ [Fig. 1(c)]. The intermediate scattering functions $S(q, t)$ are shown in Fig. 3 for the asymmetrical peaks. The normalized blue curve [Fig. 3(a)] is for a reduced field of $|h|=0.18$ and shows a much slower decay time of $\sim 20 \mathrm{~ns}$, with the entire skyrmion texture completely changing over this timescale. As we continue to move towards the continuous phase transition [43] at $|h|=0$, we see even slower dynamics. The beige curve [Fig. 3(b)] is the unnormalized, scaled data for the field of $235 \mathrm{mT}$, or a reduced field of $|h|=0.12$, to emphasize the lack of fluctuations, while the gray curve [Fig. 3(c)] shows the single-pulse data for the same field. Even without normalization, Fig. 3(b) clearly indicates a lack of decay, i.e., the skyrmion fluctuations occur on times much longer than the range of $\tau$ measured here [44].

\section{DISCUSSION}

Spontaneous fluctuations will relax on shorter timescales with an increase in energy $k_{B} T$, except at a second-order phase transition where the reverse effect occurs: critical slowing down. Taking the observations together, we have discovered that the stochastic behavior with increasing field is reminiscent of this slowing. However, this dramatic slowing down occurs well before the expected field, close to $H_{c}$, or $h=0$, where the state transforms to the uniformly magnetized phase in a continuous phase transition. This is summarized in the panel of Fig. 3(d), where the inverse time is plotted for the different relaxation times.

These results indicate that the skyrmion structure is likely in a constant state of fluctuation, where the lattice does not freeze for any applied fields. Previous work showed that a skyrmion lattice phase was stable using the saddle point approximation-which is valid for small fluctuation corrections to the mean-field value of the order parameter [45]. The present work shows that this assumption may not be the case, however. We conclude that while the average order parameter is prominent in this phase leading to a stable lattice phase, so are the fluctuations, which can completely wash out the order temporarily in some regions [see Fig. 3(a)], indicating a significant role of fluctuations in this phase. This implies that small fluctuation corrections to the mean-field theory may not be justified and would not be able to reproduce this considerable effect.

This can be further illustrated quantitatively by calculating the temperature region where fluctuations dominate. Using the Ginzburg-Landau theory of phase transitions as a model, the importance of fluctuations near a critical point can be captured by the Levanyuk-Ginzburg criterion in $d$ dimensions $[46,47]$,

$$
\tau_{R} \ll \tau_{\mathrm{LG}} \sim\left[\left(\frac{\xi}{a}\right)^{d} \frac{\Delta C}{k_{B}}\right]^{\frac{d-4}{2}},
$$

for the reduced temperature $\tau_{R}=\left(T-T_{c}\right) / T_{c}$. Since we are using a magnetic field to induce the phase transformation, we can estimate this with the reduced field $h$ without loss of generality. Careful heat capacity measurements have been carried out on similar skyrmion systems, reporting $\Delta C$ per $k_{B}$ 

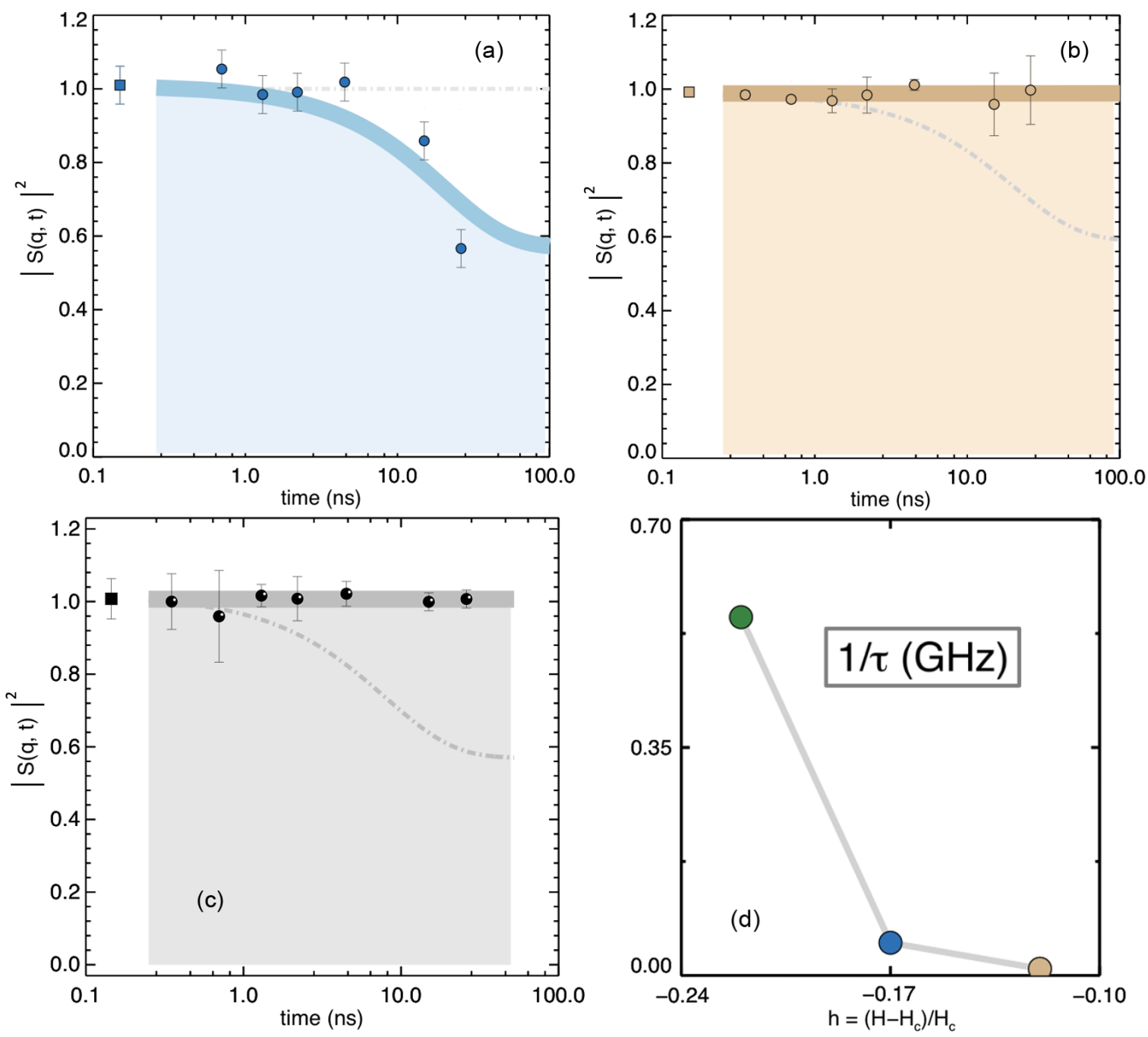

FIG. 3. Intermediate scattering functions for skyrmion fluctuations. (a) $S(q, t)$ for a magnetic field of $|h|=0.18$ (blue) shows dynamics with a relaxation time of $\sim 20 \mathrm{~ns}$ with a fit to exponential behavior. (b) $S(q, t)$ for skyrmion fluctuations at $|h|=0.12$ (beige) shows no fluctuations in the observed range, with the gray dashed line indicating the $|h|=0.18$ data [also plotted in (c)] for comparison. (c) $S(q, t)$, or effectively $S(q, 0)$, for the same phase region at $|h|=0.12$, which is defined by single-shot data. For this curve, since single-shot data are inherently $t=0$, the time axis represents the machine configuration of the electron accelerator for producing the time delay which was used when single shots were filtered from the two-pulse data. This single-pulse curve is used for normalizing any differences due to coherence or machine parameter changes with time delay. For all $S(q, t)$ curves in (a)-(c), single-pulse data were also collected for a given magnetic field, and plotted as square symbols broken from the rest of the curve as a guide to the eye. (d) The timescales $1 / \tau$ of the skyrmion phase in units of GHz plotted with reduced field $h$. The colored symbols correspond to the data plotted with the same color in Figs. 2 and 3 , as well as with the field points in Fig. 1(c).

values of about 1.8-2.2 [43]. Though this is for a different skyrmion lattice, it gives us an estimate to within an order of magnitude. This, taken together with the $d=2$ designation for this system, we can estimate $h_{\mathrm{LG}} \sim 10^{-3}-10^{-2}$, shown in the inset of Fig. 1(c) (blue area). This Ginzburg region, defined as where the fluctuations cause the breakdown of Ginzburg-Landau theory, is estimated to be much smaller then our observations indicate.

The Levanyuk-Ginzburg criterion is well-known to be a good description in systems such as BCS superconductors, but breaks down for the high- $T_{c}$ systems due to a substantially reduced pair correlation length [48]. Since the estimated Ginzburg region of the skyrmion lattice phase is very close to $h=0$, the typical method of handling fluctuations may not apply to the skyrmion lattice state. The standard procedure is to include corrections that involve quadratic gradient terms only, if inhomogeneities are small. Our work suggests that this description cannot be rectified by simply adding higherorder gradient terms to the Ginzburg-Landau theory. In other words, the breakdown is not only due to the limited correlation length as seen in the high- $T_{c}$ superconductors, but may have additional analogous behavior to more fundamental physics in these systems, such as fluctuation-induced phase instabilities. For instance, the topological nature could be playing a role in the stochastic behavior of the skyrmion lattice. Clearly, the severity of the measured fluctuations are such that the phenomenological Ginzburg-Landau theory does not capture this, and thus a more advanced theoretical treatment may 
be required to understand this type of phase transition for a topological state.

\section{CONCLUSIONS}

In summary, we have measured the spontaneous fluctuations of the skyrmion lattice state as a function of magnetic field. We observe a low-energy, underdamped excitation of this lattice, which occurs on a nanosecond timescale and provides insight into how the phase forms and how skyrmion lattice interactions, and skyrmion-skyrmion pair interactions, couple. We found the state to exhibit fluctuations which slow down near the magnetic-field-induced critical point, suggesting an inability for the system to energetically favor freezing over the fields measured. The breakdown of Ginzburg-Landau mean-field theory is prominent and seems to occur throughout the entire phase here and points to the concept that topological phase objects may have more in common with high-temperature superconductors. This is not only due to the shorter correlation length however, but to the fact that both are heavily dependent on the the appearance of dynamic fluctuations in low dimensions.

\section{ACKNOWLEDGMENTS}

We would like to acknowledge helpful discussions with $\mathrm{M}$. E. Peskin and J. W. Goodman. Special thanks to C. C. Turner for help with a calculation in the computation of $h_{\mathrm{LG}}$. We also gratefully acknowledge support from K. DeMarco. This work is supported by the U.S. Department of Energy, Office of Science, Basic Energy Sciences, Materials Sciences and Engineering Division, under Contract No. DE-AC02-76SF00515. The use of the Linac Coherent Light Source (LCLS), SLAC National Accelerator Laboratory, is supported by the U.S. Department of Energy, Office of Science, Office of Basic Energy Sciences under Contract No. DE-AC02-76SF00515. S.A.M. acknowledges support by the U.S. Office of Naval Research, In-House Lab Independent Research program. R.S., S.D.K., P.F., and S.R. acknowledge support by the U.S. Department of Energy, Office of Science, Office of Basic Energy Sciences, Materials Sciences and Engineering Division under Contract No. DE-AC02-05-CH11231 (NEMM program MSMAG). R.S. acknowledges funding from the Nebraska EPSCoR under the FIRST Award No. OIA-1557417. J.J.T. acknowledges support from the U.S. DOE, Office of Science, Basic Energy Sciences through the Early Career Research Program.
[1] A. Fert, N. Reyren, and V. Cros, Magnetic skyrmions: Advances in physics and potential applications, Nat. Rev. Mater. 2, 17031 (2017).

[2] N. Nagaosa and Y. Tokura, Topological properties and dynamics of magnetic skyrmions, Nat. Nanotechnol. 8, 899 (2013).

[3] F. Büttner, C. Moutafis, M. Schneider, B. Krüger, C. M. Günther, J. Geilhufe, C. V. K. Schmising, J. Mohanty, B. Pfau, S. Schaffert, A. Bisig, M. Foerster, T. Schulz, C. A. F. Vaz, J. H. Franken, H. J. M. Swagten, M. Kläui, and S. Eisebitt, Dynamics and inertia of skyrmionic spin structures, Nat. Phys. 11, 225 (2015).

[4] U. Ritzmann, S. von Malottki, J.-V. Kim, S. Heinze, J. Sinova, and B. Dupé, Trochoidal motion and pair generation in skyrmion and antiskyrmion dynamics under spin-orbit torques, Nat. Electron. 1, 451 (2018).

[5] S. Woo, K. M. Song, H.-S. Han, M.-S. Jung, M.-Y. Im, K.-S. Lee, K. S. Song, P. Fischer, J.-I. Hong, J. W. Choi, B.-C. Min, H. C. Koo, and J. Chang, Spin-orbit torque-driven skyrmion dynamics revealed by time-resolved x-ray microscopy, Nat. Commun. 8, 15573 (2017).

[6] W. Jiang, P. Upadhyaya, W. Zhang, G. Yu, M. B. Jungfleisch, F. Y. Fradin, J. E. Pearson, Y. Tserkovnyak, K. L. Wang, O. Heinonen et al., Blowing magnetic skyrmion bubbles, Science 349, 283 (2015).

[7] D. Capic, D. A. Garanin, and E. M. Chudnovsky, Biskyrmion lattices in centrosymmetric magnetic films, Phys. Rev. Research 1, 033011 (2019).

[8] J. C. Loudon, A. C. Twitchett-Harrison, D. Cortés-Ortuño, M. T. Birch, L. A. Turnbull, A. ŠtefančičF. Y. Ogrin, E. O. Burgos-Parra, N. Bukin, A. Laurenson, H. Popescu, M. Beg, O. Hovorka, H. Fangohr, P. A. Midgley, G. Balakrishnan, and
P. D. Hatton, Do images of biskyrmions show type-II bubbles? Adv. Mater. 31, 1806598 (2019).

[9] R. Streubel, D. S. Bouma, F. Bruni, X. Chen, P. Ercius, J. Ciston, A. T. N'Diaye, S. Roy, S. D. Kevan, P. Fischer, and F. Hellman, Magnetic materials: Chiral spin textures in amorphous iron-germanium thick films, Adv. Mater. 33, 2170061 (2021).

[10] X. Z. Yu, Y. Tokunaga, Y. Kaneko, W. Z. Zhang, K. Kimoto, Y. Matsui, Y. Taguchi, and Y. Tokura, Biskyrmion states and their current-driven motion in a layered manganite, Nat. Commun. $\mathbf{5}$, 3198 (2014).

[11] S. A. Montoya, S. Couture, J. J. Chess, J. C. T. Lee, N. Kent, D. Henze, S. K. Sinha, M.-Y. Im, S. D. Kevan, P. Fischer, B. J. McMorran, V. Lomakin, S. Roy, and E. E. Fullerton, Tailoring magnetic energies to form dipole skyrmions and skyrmion lattices, Phys. Rev. B 95, 024415 (2017).

[12] S. A. Montoya, S. Couture, J. J. Chess, J. C. T. Lee, N. Kent, M.-Y. Im, S. D. Kevan, P. Fischer, B. J. McMorran, S. Roy, V. Lomakin, and E. E. Fullerton, Resonant properties of dipole skyrmions in amorphous Fe/Gd multilayers, Phys. Rev. B 95, 224405 (2017)

[13] V. Esposito, X. Y. Zheng, M. H. Seaberg, S. A. Montoya, B. Holladay, A. H. Reid, R. Streubel, J. C. T. Lee, L. Shen, J. D. Koralek, G. Coslovich, P. Walter, S. Zohar, V. Thampy, M. F. Lin, P. Hart, K. Nakahara, P. Fischer, W. Colocho, A. Lutman et al., Skyrmion fluctuations at a first-order phase transition boundary, Appl. Phys. Lett. 116, 181901 (2020).

[14] C. Bostedt, S. Boutet, D. M. Fritz, Z. Huang, H. J. Lee, H. T. Lemke, A. Robert, W. F. Schlotter, J. J. Turner, and G. J. Williams, Linac Coherent Light Source: The first five years, Rev. Mod. Phys. 88, 015007 (2016). 
[15] F.-J. Decker, K. L. F. Bane, W. Colocho, A. A. Lutman, and J. C. Sheppard, Recent developments and plans for two bunch operation with up to $1 \mu$ s separation at LCLS, in 38th International Free Electron Laser Conference FEL2017, Santa Fe, NM, USA, TUP023 and SLAC-PUB-17128, https://www.slac. stanford.edu/pubs/slacpubs/17000/slac-pub-17128.pdf .

[16] L. Shen, M. Seaberg, E. Blackburn, and J. J. Turner, A snapshot review-fluctuations in quantum materials: From skyrmions to superconductivity, MRS Adv. 6, 221 (2021).

[17] M. H. Seaberg, B. Holladay, J. C. T. Lee, M. Sikorski, A. H. Reid, S. A. Montoya, G. L. Dakovski, J. D. Koralek, G. Coslovich, S. Moeller, W. F. Schlotter, R. Streubel, S. D. Kevan, P. Fischer, E. E. Fullerton, J. L. Turner, F.-J. Decker, S. K. Sinha, S. Roy, and J. J. Turner, Nanosecond X-Ray Photon Correlation Spectroscopy on Magnetic Skyrmions, Phys. Rev. Lett. 119, 067403 (2017).

[18] J. C. T Lee, J. J. Chess, S. A. Montoya, X. Shi, N. Tamura, S. K. Mishra, P. Fischer, B. J. McMorran, S. K. Sinha, E. E. Fullerton et al., Synthesizing skyrmion bound pairs in Fe-Gd thin films, Appl. Phys. Lett. 109, 022402 (2016).

[19] K. Chesnel, J. J. Turner, M. Pfeifer, and S. D. Kevan, Probing complex materials with coherent soft x-rays, Appl. Phys. A 92, 431 (2008).

[20] G. L. Dakovski, P. Heimann, M. Holmes, O. Krupin, M. P. Minitti, A. Mitra, S. Moeller, M. Rowen, W. F. Schlotter, and J. J. Turner, The soft $\mathrm{x}$-ray research instrument at the Linac Coherent Light Source, J. Synchrotron Radiat. 22, 498 (2015).

[21] W. F. Schlotter, J. J. Turner, M. Rowen, P. Heimann, M. Holmes, O. Krupin, M. Messerschmidt, S. Moeller, J. Krzywinski, R. Soufli et al., The soft x-ray instrument for materials studies at the Linac Coherent Light Source x-ray free-electron laser, Rev. Sci. Instrum. 83, 043107 (2012).

[22] P. Emma, R. Akre, J. Arthur, R. Bionta, C. Bostedt, J. Bozek, A. Brachmann, P. Bucksbaum, R. Coffee, F. J. Decker et al., First lasing and operation of an ångstrom-wavelength free-electron laser, Nat. Photonics 4, 641 (2010).

[23] G. Blaj, P. Caragiulo, G. Carini, S. Carron, A. Dragone, D. Freitag, G. Haller, P. Hart, J. Hasi, R. Herbst et al., X-ray detectors at the Linac Coherent Light Source, J. Synchrotron Radiat. 22, 577 (2015).

[24] L. Strüder, S. Eppa, D. Rolles, R. Hartmann, P. Holl, G. Lutz, H. Soltau, R. Eckart, C. Reich, K. Heinzinger et al., Large-format, high-speed, x-ray pnCCDs combined with electron and ion imaging spectrometers in a multipurpose chamber for experiments at 4th generation light sources, Nucl. Instrum. Methods Phys. Res., Sect. A 614, 483 (2010).

[25] P. Heimann, O. Krupin, W. F. Schlotter, J. Turner, J. Krzywinski, F. Sorgenfrei, M. Messerschmidt, D. Bernstein, J. Chalupsky, V. Hajkova et al., Linac Coherent Light Source soft x-ray materials science instrument optical design and monochromator commissioning, Rev. Sci. Instrum. 82, 093104 (2011).

[26] K. Tiedtke, A. A. Sorokin, U. Jastrow, P. Juranic, S. Kreis, N. Gerken, M. Richter, U. Arp, Y. Feng, D. Nordlund et al., Absolute pulse energy measurements of soft X-rays at the Linac Coherent Light Source, Opt. Express 22, 21214 (2014).

[27] S. Moeller, G. Brown, G. Dakovski, B. Hill, M. Holmes, J. Loos, R. Maida, E. Paiser, W. Schlotter, J. J. Turner et al., Pulse energy measurement at the SXR instrument, J. Synchrotron Radiat. 22, 606 (2015).

[28] J. Chalupsky, P. Bohacek, V. Hajkova, S. P. Hau-Riege, P. A. Heimann, L. Juha, J. Krzywinski, M. Messerschmidt, S. P. Moeller, B. Nagler et al., Comparing different approaches to characterization of focused x-ray laser beams, Nucl. Instrum. Methods Phys. Res., Sect. A 631, 130 (2011).

[29] J. Chalupský, P. Boháček, T. Burian, V. Hájková, S. P. Hau-Riege, P. A. Heimann, L. Juha, M. Messerschmidt, S. P. Moeller, B. Nagler, M. Rowen, W. F. Schlotter, M. L. Swiggers, J. J. Turner, and J. Krzywinski, Imprinting a Focused X-ray Laser Beam to Measure Its Full Spatial Characteristics, Phys. Rev. Appl. 4, 014004 (2015).

[30] A. Singh, J. C. T. Lee, K. E. Avila, Y. Chen, S. A. Montoya, E. E. Fullerton, P. Fischer, K. A. Dahmen, S. D. Kevan, M. K. Sanyal, and S. Roy, Scaling of domain cascades in stripe and skyrmion phases, Nat. Commun. 10, 1988 (2019).

[31] J. W. Goodman, Speckle Phenomena in Optics: Theory and Applications (Roberts \& Company, Englewood, CO, 2007).

[32] C. Gutt, L. M. Stadler, A. Duri, T. Autenrieth, O. Leupold, Y. Chushkin, and G. Grübel, Measuring temporal speckle correlations at ultrafast $\mathrm{x}$-ray sources, Opt. Express 17, 55 (2009).

[33] S. O. Hruszkewycz, M. Sutton, P. H. Fuoss, B. Adams, S. Rosenkranz, K. F. Ludwig, Jr., W. Roseker, D. Fritz, M. Cammarata, D. Zhu, S. Lee, H. Lemke, C. Gutt, A. Robert, G. Grübel, and G. B. Stephenson, High Contrast X-Ray Speckle from Atomic-Scale Order in Liquids and Glasses, Phys. Rev. Lett. 109, 185502 (2012).

[34] A. J. F. Siegert, On the fluctuations in signals returned by many independently moving scatterers, MIT Radiation Laboratory Report No. 465 (1943).

[35] B. J. Berne and R. Pecora, Dynamic Light Scattering: With Applications to Chemistry, Biology, and Physics (Dover, Mineola, NY, 2000).

[36] L. Zhao, Z. Wang, X. Zhang, X. Liang, J. Xia, K. Wu, H.-A. Zhou, Y. Dong, G. Yu, K. L. Wang, X. Liu, Y. Zhou, and W. Jiang, Topology-Dependent Brownian Gyromotion of a Single Skyrmion, Phys. Rev. Lett. 125, 027206 (2020).

[37] J.-J. Song, R. Bhattacharya, H. Kim, J. Chang, T.-Y. Tang, H. Guo, S. K. Ghosh, Y. Yang, Z. Jiang, H. Kim, T. P. Russell, G. Arya, S. Narayanan, and S. K. Sinha, One-Dimensional Anomalous Diffusion of Gold Nanoparticles in a Polymer Melt, Phys. Rev. Lett. 122, 107802 (2019).

[38] Y. Onose, Y. Okamura, S. Seki, S. Ishiwata, and Y. Tokura, Observation of Magnetic Excitations of Skyrmion Crystal in a Helimagnetic Insulator $\mathrm{Cu}_{2} \mathrm{OSeO}_{3}$, Phys. Rev. Lett. 109, 037603 (2012).

[39] M. Mochizuki, Spin-Wave Modes and their Intense Excitation Effects in Skyrmion Crystals, Phys. Rev. Lett. 108, 017601 (2012).

[40] Y. Okamura, F. Kagawa, M. Mochizuki, M. Kubota, S. Seki, S. Ishiwata, M. Kawasaki, Y. Onose, and Y. Tokura, Microwave magnetoelectric effect via skyrmion resonance modes in a helimagnetic multiferroic, Nat. Commun. 4, 2391 (2013).

[41] O. Petrova and O. Tchernyshyov, Spin waves in a skyrmion crystal, Phys. Rev. B 84, 214433 (2011). 
[42] J. Zang, M. Mostovoy, J. H. Han, and N. Nagaosa, Dynamics of Skyrmion Crystals in Metallic Thin Films, Phys. Rev. Lett. 107, 136804 (2011).

[43] A. Bauer, M. Garst, and C. Pfleiderer, Specific Heat of the Skyrmion Lattice Phase and Field-Induced Tricritical Point in MnSi, Phys. Rev. Lett. 110, 177207 (2013).

[44] Importantly, this "static" two-pulse curve also confirms that the system is not being pumped by the first pulse for our experimental parameters and that there are no changes due to the different electron accelerator configurations used to produce the various time separations. This provides further evidence that we are not inducing excitations in the system by the first pulse, in addition to Fig. 2(b). The pulse energy for these measurements was less than $3 \mu \mathrm{J}$, well over two orders of magnitude below the calculated damage threshold.

[45] S. Mühlbauer, B. Binz, F. Jonietz, C. Pfleiderer, A. Rosch, A. Neubauer, R. Georgii, and P. Böni, Skyrmion lattice in a chiral magnet, Science 323, 915 (2009).

[46] A. P. Levanyuk, Contribution to the theory of light scattering near the second-order phase-transition points, Zh. Eksp. Teor Fiz. 36, 810 (1959) [Sov. Phys. JETP 36, 571 (1959)].

[47] V. L. Ginzburg, Some remarks on phase transitions of the second kind and the microscopic theory of ferroelectric materials, Fiz. Tverd. Tela 2, 2031 (1960) [Sov. Phys. Solid State 2, 1824 (1960)].

[48] P. C. Hohenberg and A. P. Krekhov, An introduction to the Ginzburg-Landau theory of phase transitions and nonequilibrium patterns, Phys. Rep. 572, 1 (2015). 